\title{
Review: Precision livestock farming: building 'digital representations' to bring the animals closer to the farmer
}

\author{
T. Norton ${ }^{1 \dagger} \odot$, C. Chen ${ }^{1}$, M. L. V. Larsen ${ }^{1,2}$ and D. Berckmans $s^{1,3}$ \\ ${ }^{1}$ M3 BIORES - Measure, Model and Manage Bioresponses, Division Animal and Human Health Engineering, Department of Biosystems, KU Leuven, Kasteelpark Arenberg \\ 30, B-3001 Leuven, Belgium; '2Department of Animal Science, Aarhus University, Blichers Allé 20, 8830 Tjele, Denmark; ${ }^{3}$ BioRICS NV, Technologielaan 3, 3001 Leuven, \\ Belgium
}

(Received 26 May 2019; Accepted 29 July 2019; First published online 13 September 2019)

\begin{abstract}
Economic pressures continue to mount on modern-day livestock farmers, forcing them to increase herds sizes in order to be commercially viable. The natural consequence of this is to drive the farmer and the animal further apart. However, closer attention to the animal not only positively impacts animal welfare and health but can also increase the capacity of the farmer to achieve a more sustainable production. State-of-the-art precision livestock farming (PLF) technology is one such means of bringing the animals closer to the farmer in the facing of expanding systems. Contrary to some current opinions, it can offer an alternative philosophy to 'farming by numbers'. This review addresses the key technology-oriented approaches to monitor animals and demonstrates how image and sound analyses can be used to build 'digital representations' of animals by giving an overview of some of the core concepts of PLF tool development and value discovery during PLF implementation. The key to developing such a representation is by measuring important behaviours and events in the livestock buildings. The application of image and sound can realise more advanced applications and has enormous potential in the industry. In the end, the importance lies in the accuracy of the developed PLF applications in the commercial farming system as this will also make the farmer embrace the technological development and ensure progress within the PLF field in favour of the livestock animals and their well-being.
\end{abstract}

Keywords: livestock production, image analysis, sound analysis, technology

\section{Implications}

This work address the main approaches utilised in developing precision livestock farming tools. Precision livestock farming is an approach that enables the farmer with more objective information about the animal to make better choices about the sustainability of their production system. This paper demonstrates some of the key solutions and the approaches taken to develop technologies with sound and image analyses.

\section{Introduction}

Economic pressures continue to mount on modern-day livestock farmers. Most farmers now find themselves in a situation where they, in order to maintain their livelihood, must exploit the economies of scale. As a result, those who are surviving often have limited time to interact with their animals. Meanwhile, attention to animal welfare has heightened and stakeholders in the livestock sector are more conscious about managing and slaughtering animals in more welfare-friendly ways

\footnotetext{
${ }^{\dagger}$ E-mail: tomas.norton@kuleuven.be
}

(Blokhuis et al., 2019). Society in general is demanding closer attention to the needs of individual animal. Thus, for the case of the most intensive livestock sectors, and especially for poultry and pig production (where a large number of animals per farm is involved), the gap between societal and production demands is widening. However, most of the society do not realise that taking good care of the animals is essential to achieve good productivity, health and welfare. Closer attention to the individual animal's needs does impact not only animal welfare and health but also the capacity of the farmer to achieve sustainability (economic, environmental and societal) targets.

Technology, which is developing at a rapid pace, is enabling a better interaction between animal and farmer despite the challenges faced. Twenty years ago, the ability to carry a personal computer was a novel experience, yet nowadays we have more powerful devices that fit in the pocket. This evolution in the information and communication technologies (ICTs) has had a significant impact on the agricultural industry too. Crop and horticultural production have for the last number of years witnessed an explosion of new software systems, monitoring devices and machines that exploit 


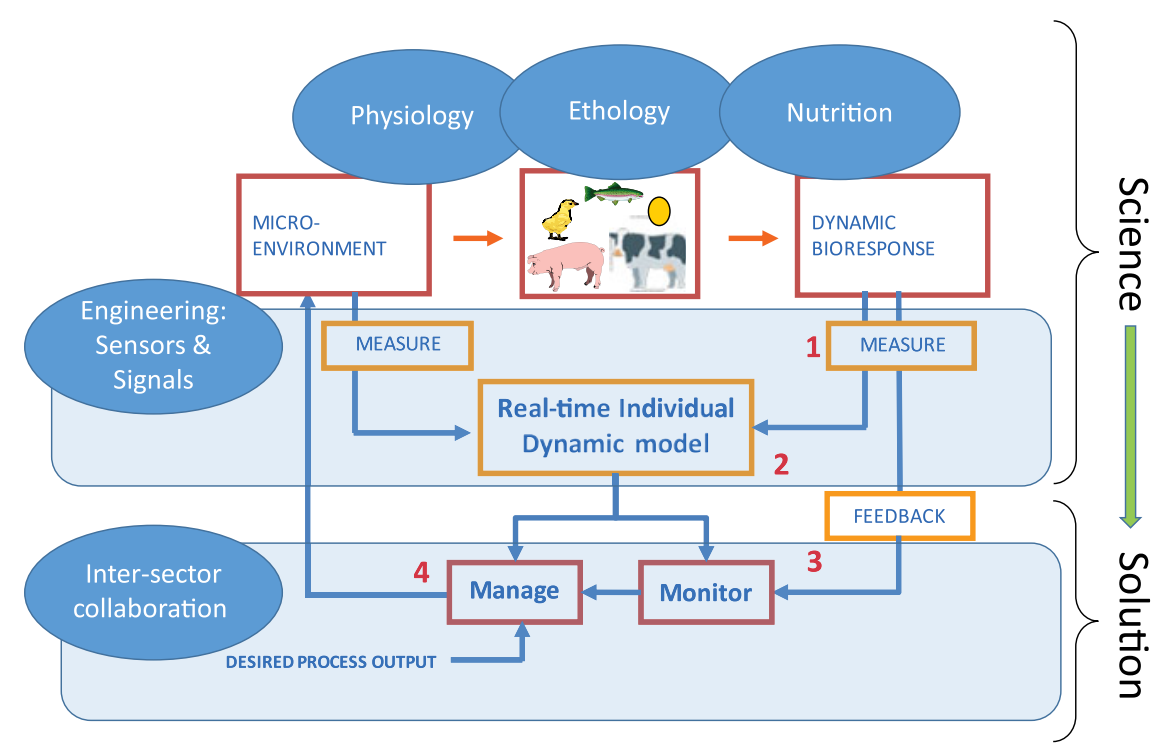

Figure 1 (Colour online) General scheme showing how bio-response monitoring and management of livestock animals can go from science to solutions (Aerts et al., 2019).

the latest capabilities in sensing, communication, processing and power management (van Evert et al., 2017). Since the early 1990s, livestock production has also started to see more research into ICT-supported management of livestock farming systems (Halachmi and Guarino, 2016). Much of the initial developments focused on decision support systems, that is, to simulate many scenarios that affect the economic or sustainability indicators of production. These optimised functions were representative of farm processes and did not seek to interface with the animals themselves. However, the animal is the central part of the process and the technology must support them at every moment to realise a better life for them, as it does for humans.

State-of-the-art technology can bring the animals closer to the farmer. Precision livestock farming (PLF) was developed to provide better information to the farmer on the animals by exploiting the known principles of process engineering to provide a level of automation. Indeed, it seems from recent contributions that PLF is the main force behind industrialising farming (Werkheiser, 2018). However, it is in fact much more than that and can instead offer an alternative philosophy to 'farming by numbers', because technology can support the farmer. Technology can collect relevant information about the animals in a continuous manner and thereby build more in-depth insight into their requirements. Some animal rights proponents go against this perspective and even argue that technology actually fuels the growth of a factory-based farming industry (Stevenson, 2017). However, we must recognise that as science gains further knowledge on the care of animals, we can also build better 'digital representations' of the animals. This empowers farmers to make better choices that are not alone driven on profits but instead on the actual needs of the animals and their care at all times.

In the literature, various studies have aimed at the development of automatic monitoring systems for livestock production. Examples include monitoring drinking behaviour (Domun et al., 2019) or to detect infected coughs by sound analysis (Exadaktylos et al., 2008), assess the thermal comfort (Shao and Xin, 2008) or estimate the live weight (Wu et al., 2004) by means of image processing. Furthermore, during the last couple of years there has been a number of scientific reviews compiling the potential of PLF from the perspective of technology developments addressing key production and environmental challenges (Benjamin and Yik, 2019; Halachmi et al., 2019; Tullo et al., 2019). Other reviews have discussed the ethical concerns of eroding the relationship between the farmer and the animal that PLF could promote (Werkheiser, 2018; Bos et al., 2018). This review will address the key approaches to monitor animals and highlight methods towards building 'digital representations' of them by giving an overview of some of the key concepts related to PLF, tool development within image and sound analyses and value discovery during PLF implementation. This review will mainly focus on the examples within the pig and poultry production.

\section{The principles of precision livestock farming}

The primary objective of PLF is to develop livestock management and monitoring systems with technologies to support the farmer (Berckmans, 2014). This includes the use of sensor technology for observing animals (Darr and Epperson, 2009), the application of modern control theory to improve autonomy of the production process (Frost et al., 2004), and the use of advanced data processing methods to synthesise and combine different types of data (Terrasson et al., 2016). Precision livestock farming is based on the interaction between different scientific disciplines and stakeholders in the livestock industry. From Figure 1 it is clear that three distinct conditions within a system need to be fulfilled to 


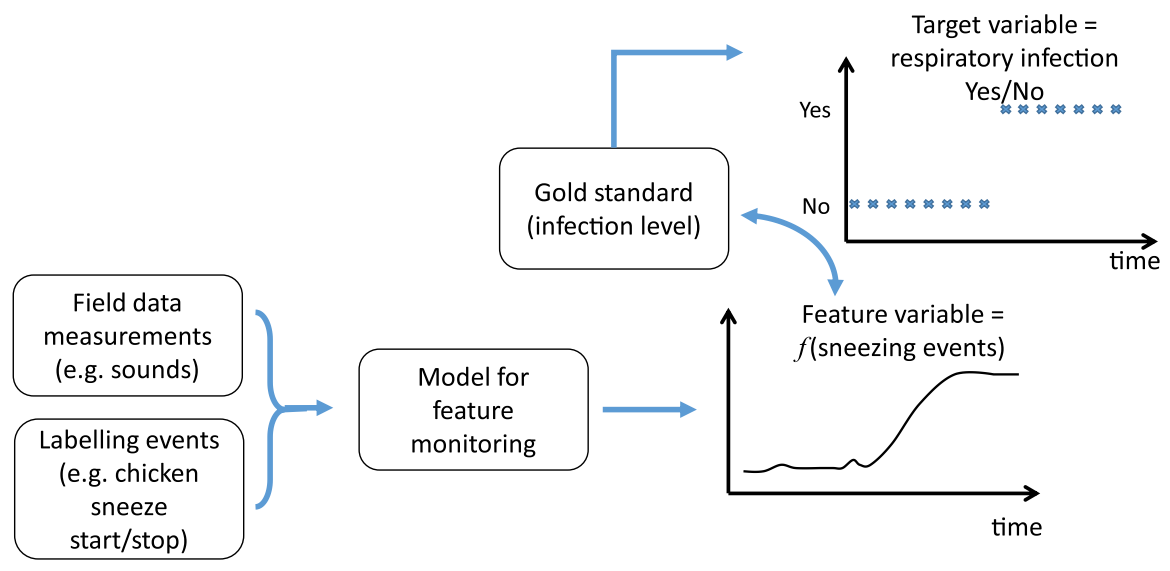

Figure 2 (Colour online) Approach for detection of chicken sneezing events from sound data (adapted from Berckmans (2013)).

achieve sufficient levels of monitoring and management to be considered a PLF system (Berckmans, 2006):

1. Animal variables (i.e. parameters related to the behavioural or physiological state of the animal) need to be measured continuously with accurate and cost-effective sensor technology,

2. A reliable prediction (expectation) must be available on how animal variables will vary or how the animal will respond at every moment, and

3. Predictions and on-line measurements are integrated in an analysing algorithm for automatic monitoring and/or management.

It should be noted that the development of the PLF systems require collaboration among different disciplines. An example of the collaborative PLF methodology (Carpentier et al., 2019) is the development of a system for detection of an occurring chicken respiratory infection (i.e., the target variable), by deriving the amount of coughs over a certain period of time (i.e. the feature variable) from a continuously measured sound signal (i.e. the process output). This methodology is further schematically illustrated in Figure 2. As can be noticed, the scheme also contains the use of gold standards to unambiguously establish the relationship between target and feature variables as well as an audiovisual labelling process. The latter is involved in identifying a number of predefined 'features' (e.g. the exact start and end point of each sneeze) from the audio and video recordings. Thus, the PLF methodology demands a high level of collaboration between multiple research fields including animal scientists (e.g. physiologists, ethologists and nutritionists), laboratory technicians, data scientists and engineers among others.

\section{Monitoring animal variables}

As described above, a PLF system includes the monitoring of animal variables, which can be, for example, behavioural or physiological measures. In the following, the review will describe in more detail how such animal variables can be continuously monitored non-invasively using examples from image and sound analyses.

\section{Image analysis}

Image analysis has been demonstrated to have potential in monitoring livestock animals since the early 1990s (van der Stuyft et al., 1991). Cameras have the advantage that they do not need to be mounted to the animal itself and, therefore, no extra stress for the animals is induced. Image analysis has been used in the past to measure some important bioresponses regarding health, welfare and growth parameters of the animals including weight estimation (Schofield et al., 1999; Mollah et al., 2010), assessing the gait and lameness of broiler chickens (Aydin et al., 2010, 2013), measuring the water intake in pigs (Kashiha et al., 2013a) and identifying marked pigs in a pen (Kashiha et al., 2013b). In the following, aggression among pigs will be used as an example of PLF research using image analysis.

Aggression among pigs as an example. A key welfarefocused application of image analysis in the PLF research is to analyse the social interactions of animals. An example of such is aggression among pigs. It is widely recognised that aggression is an important problem that threatens the health, welfare and growth of pigs in modern pig industry ( $D^{\prime}$ Eath and Turner, 2009). Usually, aggressive behaviours of pigs are found by direct observation of the producer. However, manual observation is labour-intensive and time-consuming. Detection of such problems with computer vision technologies and image analysis can instead provide advantages of uninterrupted, real-time and continuous monitoring. Using this technology for recognition and control of aggression can help to improve the efficiency of recognition, increase animal welfare and productivity and reduce economic losses of pig farms (Faucitano, 2001; Bracke et al., 2002). Recently, image analysis has been widely used for behaviour analysis of pigs including tripping and stepping behaviour recognition (Gronskyte et al., 2015) and mounting behaviour recognition (Nasirahmadi et al., 2016). However, as the complexity of aggressive behaviours in pigs is very high, the investigation of aggressive behaviours in pigs based on image analysis has been limited. Viazzi et al. (2014) focused on the detection of aggressive behaviour among pigs in general, whereas 
Oczak et al. (2014) and Chen et al. (2017) classified the aggressive behaviour into high- and medium-aggression among pigs. However, only using the high and medium intensity for definition and recognition of aggressive behaviours will be subject to greater interference by other behaviours. For instance, chase and play among pigs can also produce similar high intensities (Viazzi et al., 2014). Thus, more work is needed to be able to recognise the unwanted behavioural trait, aggression, from the wanted behavioural trait, play, using image analysis. One approach to achieve this distinction would be the ability to recognise specific aggressive markers from image analysis, including the head-to-head knocking, head-to-body knocking, parallel pressing, inverse parallel pressing, ear biting, neck biting, body biting and tail biting (Oczak et al., 2013).

A PLF tool that recognises specific behavioural patterns, such as the aggressive behaviour markers of pigs, on the individual animal level by image analysis uses several steps and techniques that is described in the following, including target tracking, extraction of animal foreground, extraction of behavioural features in the data and recognition of important behavioural interactions.

Target tracking. To recognise specific behavioural patterns on the individual level using image analysis first demands a technique that can individually recognise each animal from the other animals in the group. This is also referred to as target tracking. However, presently many computer vision systems have the shortcoming that they cannot perform this task continuously without labelling the animals. Even with manual video recordings it is not easy to assign an identity to each animal and keep track (Oczak et al., 2014). Previously, the solution to this problem was either to evaluate the behaviour at the group level (Viazzi et al., 2014) or to mark individuals with artificial symbols (Kashiha et al., 2013b). However, neither of these methods meets the requirements of commercial farms for individual selection of animals. Methods currently being investigated as alternatives to manual labelling include the 5D Gaussian model (Ahrendt et al., 2011) and the Gabor texture feature method (Huang et al., 2018).

Extraction of animal foreground. A second step is to be able to detect the animals from other objects in the environment, also referred to extraction of animal foreground or image segmentation. After such a procedure, the image should only include the animals and the rest as a unified background colour. Some methods can extract animal foreground with high accuracy but cannot separate animals that are close together (target adhesion) and, thus, make it difficult to subsequently locate the feature points on each animal body (e.g. the mixed Gauss model method (Guo et al., 2014)). Other methods can separate animals that are close together, but cannot be used for accurate extraction of animal foreground as the contour of the animal is either rough and incomplete or of a specific shape (e.g. the Otsu-based background subtraction proposed by Nasirahmadi et al. (2015) and the method of merging fitted ellipses proposed by Lu et al. (2016)). Thus, more work is required on this challenge.

Extraction of behavioural features in the data. A third step is to decide on the behavioural features that should be extracted to recognise the behaviour in question and to develop techniques to extract these chosen behavioural features. This is a part of the image analysis process that needs considerable consideration. In the case of pig aggression, features of mean intensity and occupation index (Viazzi et al., 2014; Oczak et al., 2014) were found to be useful in the past. Later, the acceleration feature (Chen et al., 2017) and motion features with higher discrimination (e.g. kinetic energy (Zhao et al., 2016), displacement, etc.) have been applied with success and can be further developed and combined with the position features of aggressive pigs (e.g. distance between head and head, distance between head and body and distance between head and tail). In the process of behavioural feature extraction, the difficulty lies in the location of individual feature points on the animal body including, for example, the location of the animals head and tail (Kashiha et al., 2013a). Without going into further detail, existing methods for locating feature points include the point distribution model (Cangar et al., 2008) and the kink points method (proposed by Frost et al., 2004). By analysing the motion of these feature points between adjacent image frames, more accurate motion features and position features can be extracted.

Recognition of important behavioural interactions. A fourth and final step is to classify the image frames into the important behavioural interactions in question (such as aggressive behavioural markers among pigs) based on the values of the extracted behavioural features. Methods for such classifications include linear discriminant analysis (Viazzi et al., 2014), neural networks (Oczak et al., 2014) and hierarchical clustering (Chen et al., 2017), among others. The threshold of each behavioural feature can then be used in the recognition rules of, for example, aggressive behaviours. As an example, when two pigs in a frame simultaneously meet at a certain acceleration (motion feature) and with a certain distance (position feature), it can be considered as a frame with head-to-head knocking. When the ratio of such frames in a period of time exceeds a fixed value, also referred to as the minimum recognition unit (Chen et al., 2017), it is considered that an event of head-to-head knocking occurred and, thus, that an aggressive interaction among the pigs in the group occurred. Further work is required to develop better rules (thresholds) to recognise each aggressive behaviour in frames of videos and to choose the accuracy, sensitivity and specificity being suitable for evaluation of the recognition results (Oczak et al., 2014).

To summarise, image analysis do show great potential as a method to recognise specific behavioural patterns, both for the individual animal and for interactions between animals. However, challenges still exist within each step of the image analysis process that needs to be investigated further. 


\section{Sound analysis}

Sound analysis is a second method for automatic continuous recording of animal variables that is non-invasive to the animal, as the microphones measuring the sound can be mounted within the animal house without causing additional stress to the animal. Within the PLF research field and sound analysis, quite a lot of attention has been given to measuring animal vocalisation. In the following, examples of PLF research within pig and chicken vocalisations will be presented.

Pig vocalisations. Various studies comprise automatic detection of pig coughs, a good indicator for respiratory problems in pigs. This automatic cough detection is not new. In 1999, Van Hirtum et al. published on selecting coughs (Van Hirtum et al., 1999). In the years following, the detection algorithm was further improved (Van Hirtum and Berckmans, 2003) and a recent study of Berckmans et al. (2015) showed that a detection tool could give warnings up to 2 weeks earlier, compared to a situation where the pigs were observed by the farmer and the veterinarian. In addition to the cough detection, Van Hirtum and Berckmans (2002), Exadaktylos et al. (2008) and Ferrari et al. (2008) showed that it is possible to decipher between cough from healthy and sick pigs. Van Hirtum and Berckmans (2003) investigated the possibility to use cough sound as a biomarker for three types of aerial pollution (irritating gas, dust and temperature). Thorough analysis of pig vocalisations including duration and amplitude of the vocalisation signal can also be used as a sign of distress in the pig (Cordeiro et al., 2018). Further classification of the pig vocalisations into vocal types including pig screams also showed to contain valuable information in identifying painful and distressful situations such as castration and ear biting (Von Borell et al., 2009; Diana et al., 2019), whereas pig barks may contain valuable information in identifying play behaviour or as alarm signals (Newberry et al., 1988; Chan et al., 2011). Recent research show a potential to differentiate pig vocalisation not only into coughs but also into screams, grunts and squeals based on several sound signal features (Vandermeulen et al., 2015; Diana et al., 2019).

Chicken vocalisations. Over the last years, there has been an increasing interest in the analyses of chicken vocalisations, as this seems to contain a lot of information about these animals. Information that can be of great importance to the farmer, if presented in the correct way. The literature on analysis of chicken vocalisations shows enormous possibilities of this research. Vocalisations of chickens have been researched in relation to welfare (Zimmerman et al., 2000), social separation (Feltenstein et al., 2002), thermal comfort (Moura et al., 2008), feather pecking (Bright, 2008), diseases (Carpentier et al., 2019) and growth (Fontana et al., 2015). However, the potential in the use of microphones is not limited to automatic recording of vocalisations but can, for example, also be used to measure feed uptake by broilers using the pecking sound (Aydin et al., 2014). The unique feature in the latter case is

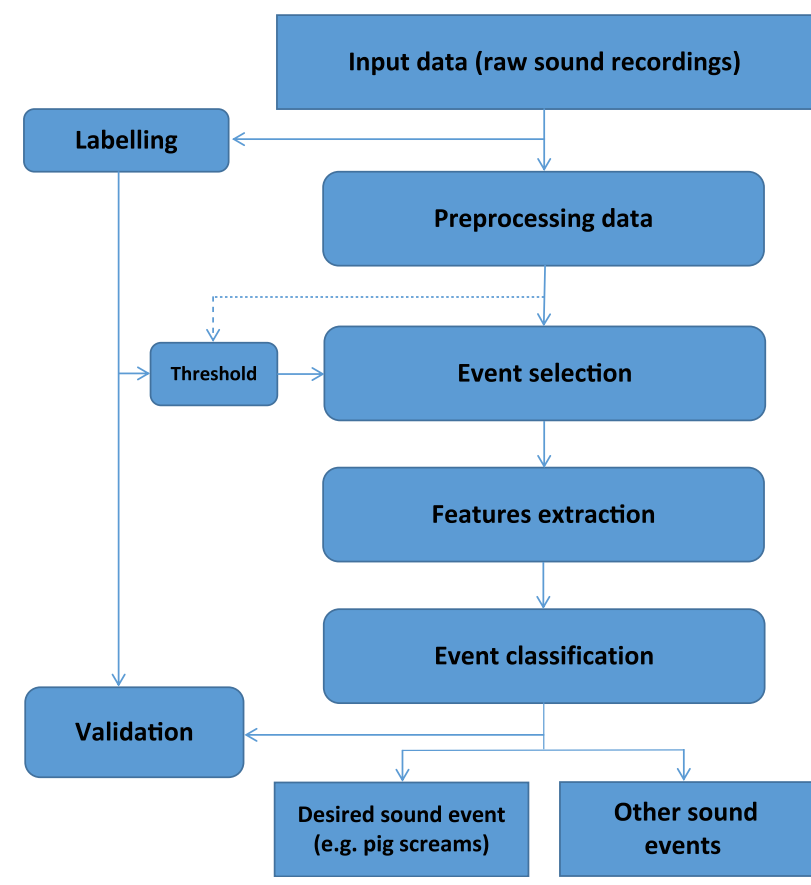

Figure 3 (Colour online) Overall approach for the development of a sound analysis-based precision livestock farming tool for livestock animals.

that a sound detection system was defined based on a sound sensor attached to the feeder pan. In this way, it was possible to perform and analyse sound measurements in real time in a fully non-invasive, but still automated, manner during the full growth process of this group of animals.

Applications to localise and monitor the health and wellbeing of livestock animals are feasible with sound analysis. For each application, the main effort is to develop an algorithm for automatic detection of sound events and then associate these specific sound events to health, distress and abnormal behaviour. As with image analysis, the workflow of such an algorithm within sound analysis takes several steps, as illustrated in Figure 3 and described in the following. These steps include sound recording, pre-processing, event selection, feature extraction and event classification.

Sound recording and pre-processing. The first step in sound analysis is the acquisition of the raw sound data (i.e. the input data). Sound is recorded and saved in blocks of a certain time, which can easily be adapted. For recording the sounds, the sampling frequency can also be adapted and the most optimal position for mounting the microphone should be explored. This includes the height of the microphone and the relative position from the walls and disruptive sound sources (e.g. ventilation). The sound data also need pre-processing, which is a specialised sound analysis procedure to separate the clear sound sources, also called foreground noises (e.g. knocking sounds, animal vocalisations), from specific interfering noises, also called background noises (fan noise, heaters). The goal is to clean up the sound data before moving on to event selection and feature extraction. For this pre-processing, many different approaches are 
possible from simple solutions like bandpass filtering to more sophisticated solutions like the ones used in speech processing. In the case of animal vocalisations, a more sophisticated solution is needed as this gives a better filtering where the background is removed as much as possible. Good filtering will make it possible to select and classify sound events with the targeted accuracy and precision and it will facilitate the next steps in the process.

Event selection and feature extraction. The third step in the algorithm workflow is to select events from the preprocessed sound data. An event is a time frame in the audio signal with a certain meaning (e.g. knocking, pecking and animal vocalisations). In this step, an automatic annotation of all relevant acoustic events is made and the events are identified with an onset and offset time. The algorithm will need a threshold or multiple thresholds to decide when an event will start or end. These thresholds are based on the labelling process that occurred during the development of the algorithm and the output of the event selection for a given threshold (i.e. how great a proportion of events were selected). The fourth step is to extract feature values from the selected events to calculate specific audio characteristics of the events including energy (total energy of the event, relation of the energy in different bands in the event), frequency information (peak frequency, mean frequency), spectral centroid, bandwidth, envelop of the event and zero-crossing rate. The main objective is to identify features with physical meaning (e.g. the mean frequency of different vocalisations).

Event classification. The final step in the algorithm workflow is the classification of the sound events. Based on the values of the different features, the events are assigned to different classes (e.g. pig vocalisations into screams and coughs). By assigning a threshold to different features, cut-offs can be made between classes. Decision on the thresholds can be done manually based on the physical meaning of a feature (e.g. a pig cough could never be shorter than $0.1 \mathrm{~s}$ or longer than $1.5 \mathrm{~s}$ ). Another approach is using automatic classification tools like hidden Markov models, Gaussian maximum likelihood estimators and neural networks among others. However, using these automatic tools makes the algorithm prone to overfitting, making it difficult or impossible to see precisely how the classification was done in retrospect. It is worth noting that these algorithms require an excellent feature database for efficient and effective classification such as databases from other audio processing disciplines (e.g. the MPEG-7 database for music processing).

To summarise, the vocalisations of livestock animals including pigs and chickens seem related to several animal health and welfare parameters. Further, sound analysis show great potential as a method to automatically recognise these specific vocalisations of livestock animals as well as to be used for other relevant applications within the livestock production.

\section{Applications of monitoring technologies in the production process}

The purpose of developing PLF applications is to monitor the health and welfare of the individual animal (and when not possible, a group of animals) in a continuous, dynamic and real-time manner on-farm to facilitate the farmer in caring for the animals. In the subsequent section, two examples of such applications from the pig and broiler production will be presented to understand the value of PLF implementation. Both application examples highlight the possibility to separate welfare monitoring from retrospective production results, so that animal health and welfare can be managed effectively in the present time.

\section{Water usage in the pig production}

During the growing process of animals, behaviours such as drinking and feeding are indicative of their health and well-being. In the production of pigs, drinking behaviour has been considered by many authors as a way to judge the health and welfare of the pigs. Pigs generally have stable diurnal drinking pattern unless influenced by stressors from disease or the environment (Madsen and Kristensen, 2005; Andersen et al., 2014). Andersen et al. (2014) found that tracking the dynamic characteristics of drinking behaviour within a day is important as part of health and welfare monitoring. The study by Madsen et al. (2005) was the first one to consider the dynamic modelling of water drinking patterns and demonstrated its power in detecting disease outbreaks (Madsen and Kristensen, 2005). One way to measure water intake is to utilise water meters, and multiple studies have found predictive information in this measure when considering unwanted events within the pig production including tail biting, pen fouling and diarrhoea (Jensen et al., 2017; Dominiak et al., 2019; Larsen et al., 2019). Other approaches focus less on the precise water consumption of the animals and instead focus more on the frequency and duration of the visits to the drinker. For example, Kashiha et al. (2013a) researched the use of camera-based monitoring of drinking behaviour in pigs. The aim of the experiment was to determine whether half-hourly water volume usage (in litres) in a pig barn could be estimated by analysing the drink nipple visits. Image analysis enabled the dynamics of water visits to be linked with meter measurements over the 13 days of the experiment. However, using water meters and cameras as above do not provide data at the individual animal level. This was enabled using high-frequency radio-frequency identification (RFID) systems that Maselyne et al. (2016) successfully implemented by comparing RFID-based visits with visual observations and flow meter measurements. All in all, the three techniques presented above provide the possibility to evaluate the time varying drinking behaviour of pigs; and given the potential RFID and camera systems, it is becoming more feasible for farmers to monitor individual water intake patterns. 


\section{Flock behaviour in broiler production}

Although not at the individual animal level, the behaviour of a broiler flock has recently been correlated to specific welfare problems in the broiler production. Fernández et al. (2018) used the commercially available PLF camera system to extract values on the activity and occupation patterns of a broiler flock. They found a positive relation between the deviations in occupation patterns and the footpad lesion scores indicating that birds, which tend to cluster together for long periods, present an increased chance of having higher levels of footpad lesions. They also found a negative relation between the deviations in the activity pattern and the hock burn scores, indicating that a higher activity of the flock would improve the hock burn scores. This can be related with the fact that having less active broiler chickens staying still for longer periods on badly conditioned litter can worsen this kind of lesion (Haslam et al., 2007). Another camera-based monitoring approach has been developed by Dawkins et al. (2013). The approach is based on an analysis approach called optical flow. This approach derives the general movement flow patterns of birds from the images, as well as the variance, skew and kurtosis of the flow and have been correlated with health and welfare indicators (Dawkins et al., 2013).

\section{The future potential of precision livestock farming in livestock production}

Welfare management is the key challenge today and often relates to both the health and production of the animals. It is not enough for the farmer to be told after the animals have left the herd that the system is not at a suitable welfare level. After this point, the farmer cannot take appropriate action. With PLF, real-time on-farm welfare monitoring and management becomes feasible, and many opportunities exist beside the examples already mentioned. Continuous monitoring of key variables on the farm will make real-time welfare management possible. As the PLF research community grows, we still have to do more work to realise the practical benefits of PLF technologies in the commercial production. Only with accurate systems can we start to convince farmers of the capacities of such technology to link productivity, health and welfare management. Examples from the pig and poultry productions have been used in the current literature review. However, the PLF field of research is most certainly not limited to these two livestock species but is also well established within, for example, the dairy and beef cattle productions. In the following, the opportunities with PLF in an often forgotten livestock production form, aquaculture, will be discussed.

Opportunities in other sectors: aquaculture as an example Aquaculture is traditionally considered a low-technology sector (Føre et al., 2018). Almost all biological responses of fish are expressed underwater. Given the industry's drive toward larger production cages, there is now an even greater need for technologies that enable farmers to maintain a high level of control over the production processes in fish cages. Fish farmers have found it hard to understand the behavioural repertoire of fish and thereby obtain direct relationship with their animals. In this context, there is a clear need for quantifying fish behaviour and translating this information into a reliable welfare assessment system. Much of the previous research has focused on a qualitative description of behaviour, whereas recent technologies coupled to stateof-the-art computer vision and machine learning techniques open the door to a data-based description of fish behaviour. This approach will allow for a more careful inspection of a species' whole behavioural repertoire, possibly highlighting patterns of behaviour that were previously overlooked by human observers. Previous research efforts aimed at aquaculture have focused on the development and use of technologies for monitoring and estimating animal variables in tanks and cages (e.g. Alfredsen et al., 2008; Føre et al., 2011). Other research has tried to link the biological responses of farmed fish with the underlying mechanisms as a function of external factors such as feeding and management (e.g. Oppedal et al., 2011). While such studies represent essential building blocks for the development of new technologybased methods for intensive aquaculture, the tools for drawing a foundation for decision support by combining monitoring technologies with knowledge on the biological characteristics of fish population has been non-existent (Føre et al., 2018). Hence, an adaptation of the methodology therein to fish farming will build a bridge between technological and biological research results and efforts.

\section{Conclusion}

Precision livestock farming can provide the key to a sustainable livestock production in the future. In this review, we have identified some of the key principles of PLF and key ideas behind the development of PLF sound and image tools as an non-invasive method of measuring animal variables. A key point is that the development and operation of PLF applications involve several steps and techniques that need to be investigated further to make the developed systems accurate and implementable in commercial environments. By adopting such tools the PLF field will progress and the farmer will also embrace the value of the technology. Therefore, we must strengthen collaboration between key stakeholders as well as ensure that scientific rigour is enforced through the development and validation of these systems.

\section{Acknowledgements}

None.

(1) T. Norton 0000-0002-0161-3189

\section{Declaration of interest}

The authors declare no conflict of interest. 
Norton, Chen and Larsen

\section{Ethics statement}

Not applicable.

\section{Software and data repository resources}

Not applicable, as no data were used and no models were created in the conduction of this literature study.

\section{References}

Aerts JM, Norton T and Berckmans D 2019. Integration of Bioresponses in management of biological processes. Course in $1^{\text {st }}$ year of Masterprogramme Biosystems Engineer, Katholieke Universiteit Leuven, pp. 360, started in 2006. Ahrendt P, Gregersen T and Karstoft H 2011. Development of a real-time computer vision system for tracking loose-housed pigs. Computers and Electronics in Agriculture 76, 169-174.

Alfredsen JA, Holand B, Solvang-Garten T and Uglem I 2008. Feeding activity and opercular pressure transients in Atlantic salmon (Salmo salar L.): application to feeding management in fish farming. Hydrobiologia 582, 199-207.

Andersen HM-L, Dybkjær L and Herskin MS 2014. Growing pigs' drinking behaviour: number of visits, duration, water intake and diurnal variation. Animal 8, 1881-1888.

Aydin A, Bahr C, Viazzi S, Exadaktylos V, Buyse J and Berckmans D 2014. A nove method to automatically measure the feed intake of broiler chickens by sound technology. Computers and Electronics in Agriculture 101, 17-23.

Aydin A, Cangar O, Ozcan SE, Bahr C and Berckmans D 2010. Application of a fully automatic analysis tool to assess the activity of broiler chickens with different gait scores. Computers and Electronics in Agriculture 73, 194-199.

Aydin A, Pluk A, Leroy T, Berckmans D and Bahr C 2013. Automatic identification of activity and spatial use of broiler chickens with different gait scores. Transactions of the ASABE 56, 1123-1132.

Benjamin M and Yik S 2019. Precision livestock farming in swine welfare: a review for swine practitioners. Animals 9, 133

Berckmans D 2006. Automatic on-line monitoring of animals by precision livestock farming. Livestock Production and Society, 287-294.

Berckmans D 2013. Basic principles of PLF: gold standard, labelling and field data. In 6th European Conference on Precision Livestock Farming (EC-PLF 2013), 10-12 September 2013, Leuven, Belgium.

Berckmans D 2014. Precision livestock farming technologies for welfare management in intensive livestock systems. Scientific and Technical Review of the Office International des Epizooties 33, 189-196.

Berckmans D, Hemeryck M, Berckmans D, Vranken E and van Waterschoot $T$ 2015. Animal sound ... talks! real-time sound analysis for health monitoring in livestock. In Proceedings of the International Symposium on Animal Environment and Welfare, 23-26 October 2015, Chongqing, China, pp. 215-222.

Blokhuis H, Veissier I, Miele M and Jones B 2019. Safeguarding farm animal welfare. In Sustainability certification schemes in the agricultural and natural resource sectors: outcomes for society and the environment (ed. M Vogt), pp. 137-154. Routledge, Taylor and Francis Group, London UK and New York, USA.

Bos JM, Bovenkerk B, Feindt PH and Van Dam YK 2018. The quantified animal: precision livestock farming and the ethical implications of objectification. Food Ethics 2, 77-92.

Bracke MB, Metz JH, Spruijt BM and Schouten WG 2002. Decision support system for overall welfare assessment in pregnant sows $B$ : validation by expert opinion. Journal of Animal Science 80, 1835-1845.

Bright A 2008. Vocalisations and acoustic parameters of flock noise from feather pecking and non-feather pecking laying flocks. British Poultry Science 49 241-249.

Cangar O, Leroy T, Guarino M, Vranken E, Fallon R, Lenehan J, Mee J and Berckmans D 2008. Automatic real-time monitoring of locomotion and posture behaviour of pregnant cows prior to calving using online image analysis Computers and Electronics in Agriculture 64, 53-60.

Carpentier L, Vranken E, Berckmans D, Paeshuyse J and Norton T 2019. Development of sound-based poultry health monitoring tool for automated sneeze detection. Computers and Electronics in Agriculture 162, 573-581.
Chan WY, Cloutier S and Newberry RC 2011. Barking pigs: differences in acoustic morphology predict juvenile responses to alarm calls. Animal Behaviour 82, 767-774.

Chen C, Zhu WX, Ma CH, Guo YZ, Huang WJ and Ruan CZ 2017. Image motion feature extraction for recognition of aggressive behaviours among group-housed pigs. Computers and Electronics in Agriculture 142, 380-387.

Cordeiro AFDS, Nääs IDA, da Silva Leitão F, de Almeida AC and de Moura DJ 2018. Use of vocalisation to identify sex, age, and distress in pig production. Biosystems Engineering 173, 57-63.

Darr $\mathrm{M}$ and Epperson W 2009. Embedded sensor technology for real time determination of animal lying time. Computers and Electronics in Agriculture 66, 106-111. Dawkins MS, Russell C, Merelie K and Roberts SJ 2013. In search of the behavioural correlates of optical flow patterns in the automated assessment of broiler chicken welfare. Applied Animal Behaviour Science 145, 44-50.

D'Eath RB and Turner SP 2009. The natural behaviour of the pig. In The welfare of pigs (ed. JN Marchant-Forde), pp. 13-45. Springer, Dordrecht, Netherlands. Diana A, Carpentier L, Piette D, Boyle LA, Berckmans D and Norton T 2019. An ethogram of biter and bitten pigs during an ear biting event: first step in the development of a precision livestock farming tool. Applied Animal Behaviour Science 215, 26-36.

Dominiak KN, Hindsborg J, Perdersen LJ and Kristensen AR 2019. Spatial modeling of pigs' drinking patterns as an alarm reducing method II. Application of a multivariate dynamic linear model. Computers and Electronics in Agriculture 161, 92-103.

Domun Y, Pedersen LJ, White D, Adeyemi 0 and Norton T 2019. Learning patterns from time-series data to discriminate predictions of tail-biting, fouling and diarrhoea in pigs. Computers and Electronics in Agriculture 163, 104878.

Exadaktylos V, Silva M, Aerts JM, Taylor CJ and Berckmans D 2008. Real-time recognition of sick pig cough sounds. Computers and Electronics in Agriculture 63, 207-214.

Faucitano L 2001. Causes of skin damage to pig carcasses. Canadian Journal of Animal Science 81, 39-45.

Feltenstein MW, Ford NG, Freeman KB and Sufka KJ 2002. Dissociation of stress behaviors in the chick social-separation-stress procedure. Physiology \& Behavior 75, 675-679.

Fernández AP, Norton T, Tullo $E$, van Hertem T, Youssef A, Exadaktylos V, Vranken E, Guarino M and Berckmans D 2018. Real-time monitoring of broiler flock's welfare status using camera-based technology. Biosystems Engineering 173, 103-114.

Ferrari S, Silva M, Guarino M, Aerts JM and Berckmans D 2008. Cough sound analysis to identify respiratory infection in pigs. Computers and Electronics in Agriculture 64, 318-325.

Fontana I, Tullo E, Butterworth A and Guarino M 2015. An innovative approach to predict the growth in intensive poultry farming. Computers and Electronics in Agriculture 119, 178-183.

Frost AR, French AP, Tillett RD, Pridmore TP and Welch SK 2004. A vision guided robot for tracking a live, loosely constrained pig. Computers and Electronics in Agriculture 44, 93-106.

Føre M, Alfredsen JA and Gronningsater A 2011. Development of two telemetrybased systems for monitoring the feeding behaviour of Atlantic salmon (Salmo salar L.) in aquaculture sea-cages. Computers and Electronics in Agriculture 76, 240-251.

Føre M, Frank K, Norton T, Svendsen E, Alfredsen JA, Dempster T, Eguiraun H, Watson W, Stahl A Sunde LM, Schellewald C, Skøien K, Alver MO and Berckmans D 2018. Precision fish farming: a new framework to improve production in aquaculture. Biosystems Engineering 173, 176-193.

Gronskyte R, Clemmensen LH, Hviid MS and Kulahci M 2015. Pig herd monitoring and undesirable tripping and stepping prevention. Computers and Electronics in Agriculture 119, 51-60.

Guo YZ, Zhu WX, Jiao PP and Chen JL 2014. Foreground detection of group-housed pigs based on the combination of mixture of Gaussians using prediction mechanism and threshold segmentation. Biosystems Engineering 125, 98-104.

Guo Y, Zhu W, Jiao P, Ma C and Yang J 2015. Multi-object extraction from topview group-housed pig images based on adaptive partitioning multilevel thresholding segmentation. Biosystems Engineering 135, 54-60.

Halachmi I and Guarino M 2016. Precision livestock farming: a 'per animal' approach using advanced monitoring technologies. Animal 10, 1482-1483. 
Halachmi I, Guarino M, Bewley J and Pastell M 2019. Smart animal agriculture: application of real-time sensors to improve animal well-being and production Annual Review of Animal Biosciences 7, 403-425.

Haslam SM, Knowles TG, Brown SN, Wilkins LJ, Kestin SC, Warriss PD and Nicol CJ 2007. Factors affecting the prevalence of foot pad dermatitis, hock burn and breast burn in broiler chicken. British Poultry Science 48, 264-275

Huang WJ, Zhu WX, Ma CH, Guo YZ and Chen C 2018. Identification of grouphoused pigs based on Gabor and Local Binary Pattern features. Biosystesms Engineering 166, 90-100.

Jensen DB, Toft $N$ and Kristensen AR 2017. A multivariate dynamic linear mode for early warnings of diarrhea and pen fouling in slaughter pigs. Computers and Electronics in Agriculture 135, 51-62.

Kashiha M, Bahr C, Haredasht Amirpour S, Ott S, Moons C, Niewold TA, Odberg FO and Berckmans D 2013a. The automatic monitoring of pigs water use by cameras. Computers and Electronics in Agriculture 90, 164-169.

Kashiha M, Bahr C, Ott S, Moons CP, Niewold TA, Ödberg FO and Berckmans D 2013b. Automatic identification of marked pigs in a pen using image pattern recognition. Computers and electronics in agriculture 93, 111-120.

Larsen MLV, Pedersen LJ and Jensen DB 2019. Prediction of tail biting events in finisher pigs from automatically recorded sensor data. Animals 9, 458.

Lu MZ, Xiong YJ, Li KQ, Liu LS, Yan L, Ding YQ, Lin XZ, Yang XJ and Shen MX 2016. An automatic splitting method for the adhesive piglets' gray scale image based on the ellipse shape feature. Computers and Electronics in Agriculture 120, 53-62.

Madsen TN, Andersen S and Kristensen AR 2005. Modelling the drinking patterns of young pigs using a state space model. Computers and Electronics in Agriculture 48, 39-61.

Madsen TN and Kristensen AR 2005. A model for monitoring the condition of young pigs by their drinking behaviour. Computers and Electronics in Agriculture $48,138-154$

Maselyne J, Adriaens I, Huybrechts T and De Ketelaere B 2016. Measuring the drinking behaviour of individual pigs housed in group using radio frequency identification (RFID). Animal 10, 1557-1566.

Mollah MBR, Hasan MA, Salam MA and Ali MA 2010. Digital image analysis to estimate the live weight of broiler. Computers and Electronics in Agriculture 72, 48-52.

Moura DJD, Nääs IDA, Alves ECDS, Carvalho TMRD, Vale MMD and Lima KAOD (2008). Noise analysis to evaluate chick thermal comfort. Scientia Agricola 65, 438-443.

Nasirahmadi A, Hensel O, Edwards SA and Sturm B 2016. Automatic detection of mounting behaviours among pigs using image analysis. Computers and Electronics in Agriculture 124, 295-302.

Nasirahmadi A, Richter U, Hensel O, Edwards S and Sturm B 2015. Using machine vision for investigation of changes in pig group lying patterns. Computers and Electronics in Agriculture 119, 184-190.

Newberry RC, Wood-Gush DGM and Hall JW 1988. Playful behaviour of piglets. Behavioural Processes 17, 205-216.

Oczak M, Ismayilova G, Costa A, Viazzi S, Sonoda LT, Fels M, Bahr C, Hartung J, Guarino M, Berckmans M and Vranken E 2013. Analysis of aggressive behaviours of pigs by automatic video recordings. Computers and Electronics in Agriculture 99, 209-217.

Oczak M, Viazzi S, Ismayilova G, Sonoda LT, Roulston N, Fels M, Bahr C, Hartung J, Guarino M, Berckmans D and Vranken E 2014. Classification of aggressive behaviour in pigs by activity index and multilayer feed forward neural network. Biosystems Engineering 119, 89-97.
Oppedal F, Dempster T and Stien LH 2011. Environmental drivers of Atlantic salmon behaviour in sea-cages: a review. Aquaculture 311, 1-18.

Schofield CP, Marchant JA, White RP, Brandl N and Wilson M 1999. Monitoring pig growth using a prototype imaging system. Journal of Agricultural Engineering Research 72, 205-210.

Shao B and Xin H 2008. A real-time computer vision assessment and control of thermal comfort for group-housed pigs. Computers and Electronics in Agriculture 62, 15-21.

Stevenson $P$ 2017. Precision livestock farming: could it drive the livestock sector in the wrong direction. In Proceedings of the 8th European Conference of Precision Livestock Farming, EC-PLF 2017, September 12-14, 2017, Nantes, France.

Terrasson G, Llaria A, Marra A \& Voaden S 2016. Accelerometer based solution for precision livestock farming: geolocation enhancement and animal activity identification. IOP Conference Series: Materials Science and Engineering 138 012004.

Tullo E, Finzi A and Guarino M 2019. Environmental impact of livestock farming and precision livestock farming as a mitigation strategy. Science of the Total Environment, 650, 2751-2760.

Vandermeulen J, Bahr C, Tullo E, Fontana I, Ott S, Kashiha M, Guarino M, Moons CPH, Tuyttens FAM, Niewold TA and Berckmans D 2015. Discerning pig screams in production environments. PLOS ONE 10, e0123111.

van der Stuyft E, Schofield CP, Randall JM, Wambacq P and Goedseels V 1991. Development and application of computer vision systems for use in livestock production. Computers and Electronics in Agriculture 6, 243-265.

van Evert FK, Fountas S, Jakovetic D, Crnojevic V, Travlos I and Kempenaar C 2017. Big data for weed control and crop protection. Weed Research 57, 218-233.

van Hirtum A, Aerts JM, Berckmans D, Moreaux B and Gustin P 1999. On-line cough recognizer system. The Journal of the Acoustical Society of America 106, 2191-2191.

van Hirtum A and Berckmans D 2002. Assessing the sound of cough towards vocality. Medical Engineering \& Physics 24, 535-540.

van Hirtum A and Berckmans D 2003. Fuzzy approach for improved recognition of citric acid induced piglet coughing from continuous registration. Journal of Sound Vibration 266, 677-686.

Viazzi S, Ismayilova G, Oczak M, Sonoda LT, Fels M, Guarino M, Vranken E, Hartung J, Bahr C and Berckmans D 2014. Image feature extraction for classification of aggressive interactions among pigs. Computers and Electronics in Agriculture 104, 57-62.

Von Borell E, Bünger B, Schmidt T and Horn T 2009. Vocal-type classification as a tool to identify stress in piglets under on-farm conditions. Animal Welfare 18, 407-416.

Werkheiser I 2018. Precision livestock farming and farmers' duties to livestock. Journal of Agricultural and Environmental Ethics 31, 181-195.

Wu JH, Tillett R, McFarlane N, Ju XY, Siebert JP and Schofield P 2004. Extracting the three- dimensional shape of live pigs using stereo photogrammetry. Computers and Electronics in Agriculture 44, 203-222.

Zhao J, Gu ZB, Shi MM, Lu HD, Li JP, Shen MW, Ye ZY and Zhu SM 2016. Spatial behavioural characteristics and statistics-based kinetic energy modeling in special behaviours detection of a shoal of fish in a recirculating aquaculture system. Computers and Electronics in Agriculture 127, 271-280.

Zimmerman PH, Koene P and van Hooff JA 2000. The vocal expression of feeding motivation and frustration in the domestic laying hen, Gallus gallus domesticus. Applied Animal Behaviour Science 69, 265-273. 